\title{
The Effect of Rice Flour for Amelioration of Sleeping in Social Isolated Mice
}

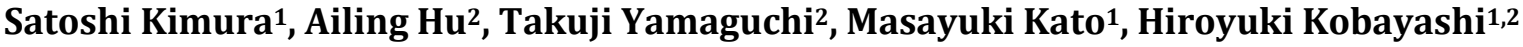 \\ ${ }^{1}$ Department of Hospital Administration, Graduate School of Medicine, Juntendo University, Tokyo, Japan \\ ${ }^{2}$ Center for Advanced Kampo Medicine and Clinical Research, Graduate School of Medicine, Juntendo University, Tokyo, Japan \\ Email: s.kimura911@gmail.com
}

How to cite this paper: Kimura, S., Hu, A., Yamaguchi, T., Kato, M. and Kobayashi, H. (2019) The Effect of Rice Flour for Amelioration of Sleeping in Social Isolated Mice. Health, 11, 464-471.

https://doi.org/10.4236/health.2019.115040

Received: March 6, 2019

Accepted: May 13, 2019

Published: May 16, 2019

Copyright $\odot 2019$ by author(s) and Scientific Research Publishing Inc. This work is licensed under the Creative Commons Attribution International License (CC BY 4.0).

http://creativecommons.org/licenses/by/4.0/

\begin{abstract}
In an era where the health-damaging effects of wheat flour (gluten) are increasingly recognized, rice flour has become an important alternative for many people, yet its psychophysiological effects remain largely unknown. Here, we report the potential beneficial effects of rice flour for sleep disturbances in stressed mice. Four-week-old male ddY mice were reared in social isolation for 4 weeks. The control group was reared in a social group. Rice flour was given orally in food to isolated mice at a dose of $2.5 \mathrm{w} / \mathrm{w} \%$ and 5.0 $\mathrm{w} / \mathrm{w} \%$ for 4 weeks from the start of isolation rearing. MF food was given to the control group. Pentobarbital-induced ( $40 \mathrm{mg} / \mathrm{kg}$, i.p.) time to sleep induction and righting reflex was measured to determine the effects of rice flour on sleep behavior. Blood samples were obtained after the experiments, and serum corticosterone was measured. Sections from the prefrontal cortex and the brainstem were isolated to measure serotonin, dopamine and interleukin (IL)-6 concentrations. We found that the administration of rice flour dose-dependently improved time to sleep and reduced sleep time in socially isolated mice. Blood corticosterone concentrations, which increased after isolation stress, were decreased after the administration of rice flour. Serotonin and dopamine concentrations in the prefrontal cortex which decreased after isolation stress improved after the administration of rice flour. Brainstem IL- 6 concentrations increased after isolation stress, but decreased dose-dependently after rice flour administration. Our results suggest that rice flour reverses sleep disturbances in mice induced by social isolation.
\end{abstract}

\section{Keywords}

Rice Flour, Isolation Stress, Sleeping Serotonin, Dopamine, Interleukin (IL)-6

\section{Introduction}

Modern life in Japan has become synonymous with stress and allergy. There is an 
alarming increase in gluten allergy, with recent data showing that approximately one third of babies and infants develop gluten allergy/sensitivity/intolerance, and $14 \%$ of people in their 20 's and over have gluten allergy. Wheat allergy is increasingly known to be one of the reasons causing stress to people in this current Japanese society. Rice flour comes up as a substitute for wheat, however, the construction of the framework of the scientific verification and the collection of the evidence regarding how rice flour influences a human mind and body are not conducted yet.

Stress is involved in clinical conditions of several diseases. Especially mental diseases such as adjustment disorder, depression, and chronic fatigue syndrome caused by chronic social stress are recognized as serious social problems [1].

Sleep, along with food, clothing and shelter, is the major problem for human beings to survive. Many people suffer from insomnia, which causes autonomic disorder, including depression, and other several diseases caused by the lack of sleep. Therefore, its clinical importance is extremely high. Furthermore, it is reported that the reduction of productivity caused by lack of sleep results in the economic loss. Compared to other countries, it has been clarified that not only Japanese average amount of sleep is remarkably short, but also there are a number of people suffering sleeping disorder. Circadian rhythm disorder including dyssomnia caused by forcing an irregular life style can be considered one of the reasons of lack of sleep. It has been also clarified that sleeping disorder is related to dementia and depression [2]. However, enough treatment has not been dealt with it yet. Rice has been long the staple food for Japanese, and it is the representative proteins which can be obtained safely and securely. Yoneyama and his group examined the correlation between the quality of sleep and the amount of three different GI (the index which shows glycemic index, and postprandial blood glucose level) of starch food (rice, bread, noodles) and the GI levels in a group of Japanese males and females. The result indicated that consuming enough amount of high GI food and rice was significantly related to the high quality of sleep; however, the amount of consuming bread was not related to the quality of sleep, and the amount of consuming noodles was related to low quality of sleep [3].

There are few studies of rice flour against stress. In the present study, we examined the effect of rice flour on sleeping disorder and brain monoamine, cytokine, and blood corticosterone using mice as stress models.

\section{Materials and Methods}

\subsection{Animals}

Three weeks male ddY mice were purchased from Japan SLC, Inc. (Shizuoka, Japan). After a week of habituation, the animals of the experimental group were placed individually in a transparent cage $(9 \times 13 \times 20 \mathrm{~cm})$ for 4 weeks. As the control group, 5 mice were reared together in a cage $(23 \times 31 \times 15.5 \mathrm{~cm})$. All animals were reared under the condition of $23^{\circ} \mathrm{C} \pm 2^{\circ} \mathrm{C}$, relative humidity $55 \% \pm$ 
10\%, 12-hour light and dark cycle from 7:00 to 19:00, free drinking, and normal food consumption (MF, Oriental Yeast Co., Ltd., Tokyo, Japan) from the habituation period to the test period. The animal tests were conducted in accordance with the guideline of Juntendo University Medical School Animal Testing (JACUC) (registration no.1022).

\subsection{Drugs and Reagents}

Princes Road manufactured by Nippon Flour Mills Co., Ltd. was used as rice flour. Rice flour was mixed with MF food (MF, Oriental Yeast Co., Ltd., Tokyo, Japan) to make it $2.5 \mathrm{w} / \mathrm{w} \%$ and $5.0 \mathrm{w} / \mathrm{w} \%$.

\section{Experimental Designs}

\subsection{Sleep Experiment}

Rice flour was administrated orally with food at a dose of $2.5 \mathrm{w} / \mathrm{w} \%$ and 5.0 $\mathrm{w} / \mathrm{w} \%(\mathrm{n}=10$ each) to a mouse reared in isolation for 4 weeks. MF food was administrated to the control group. Their body weight was measured every week. The pentobarbital (PB:40 mg/kg, i.p.) induced time to sleep introduction and the response of regular reflection were measured to see the effect on sleep.

\subsection{The Measurement of Brain Monoamines and Interleukin} (IL) -6

After the sleep test, the frontal cortex and the brainstem were collected, frozen and preserved at $-80^{\circ} \mathrm{C}$ until the measurement. The tissue was homogenized in the buffer solution ( $0.01 \mathrm{~N}$ hydrochloric acid (Wako), $1 \mathrm{mM}$ EDTA (Sigma), 4 $\mathrm{mM}$ sodium bisulfite (Sigma), and the supernatant fluid was obtained after centrifugation $\left(15,000 \mathrm{rpm} \times 30\right.$ minutes, $\left.4^{\circ} \mathrm{C}\right)$. Dopamine (Dopamine Research RIA, Labor Diagnostika Nord, Nordhorn, Germany), serotonin ((Serotonin Research RIA, Labor Diagnostika Nord, Nordhorn, Germany), and IL-6 (mouse IL-6 Immunoassay, R\&D Systems, USA) were measured by each ELIZA Kit. The total amount of protein (BCA Protein Assay kit Pierce, Rockford, IL, USA) was then measured.

\subsection{The Measurement of Blood Serum Corticosterone}

After 4 weeks of isolated rearing, blood was collected from an artery at the back of the eye under isoflurane anesthesia, and the blood serum was obtained after centrifugation $\left(15,000 \mathrm{rpm} \times 20\right.$ minutes, $\left.4^{\circ} \mathrm{C}\right)$. The blood serum was frozen and preserved at $-20^{\circ} \mathrm{C}$ until the measurement. Blood serum corticosterone was measured by Mouse Corticosterone Kit (Assay Designs, Michigan, USA).

\section{Statistical Analyses}

All data were presented as mean \pm S.E.M. The data were analyzed with Fisher's test to evaluate differences between the administration group and the control group. $\mathrm{P}$ values of $<0.05$ were considered as statistically significant on all the tests. 


\section{Results}

\subsection{The Effect of Rice Flour on Sleeping Disorder Induced by Isolation Stress}

The effect on body weight by administrating rice flour was shown in Figure 1 . The effects of rice flour on body weight were not recognized in this data.

The effect of rice flour on the pentobarbital-induced time to sleep introduction and the sleeping time induced by socially isolated stress was shown in Figure 2. The pentobarbital-induced time to sleep introduction was significantly extended by socially isolated stress. The pentobarbital-induced sleeping time was significantly decreased by socially isolated stress. Rice flour dose-dependently and significantly shortened the time to sleep introduction induced by socially isolated stress, and dose-dependently increased the sleeping time induced by isolation stress.

\subsection{The Effect of Rice Flour on Brain Monoamine and IL-6}

The effect of rice flour on the amount of serotonin and dopamine in the frontal cortex induced by socially isolated stress was shown in Figure 3. Serotonin and dopamine in the frontal cortex decreased significantly by isolation stress. Serotonin and dopamine dose-dependently increased by administrating rice flour.

The effect of rice flour on the amount of IL- 6 in the brainstem induced by socially isolated stress was shown in Figure 4. IL-6 in the brainstem increased significantly by isolation stress. Rice flour dose-dependently and significantly decreased the increased IL-6.

\subsection{The Effect of Rice Flour on Blood Corticosterone}

The effect of rice flour on blood corticosterone induced by socially isolated stress was shown in Figure 5. The blood corticosterone increased by isolation stress. The increased corticosterone dose-dependently decreased by administrating rice flour. However, no significant differences were observed.

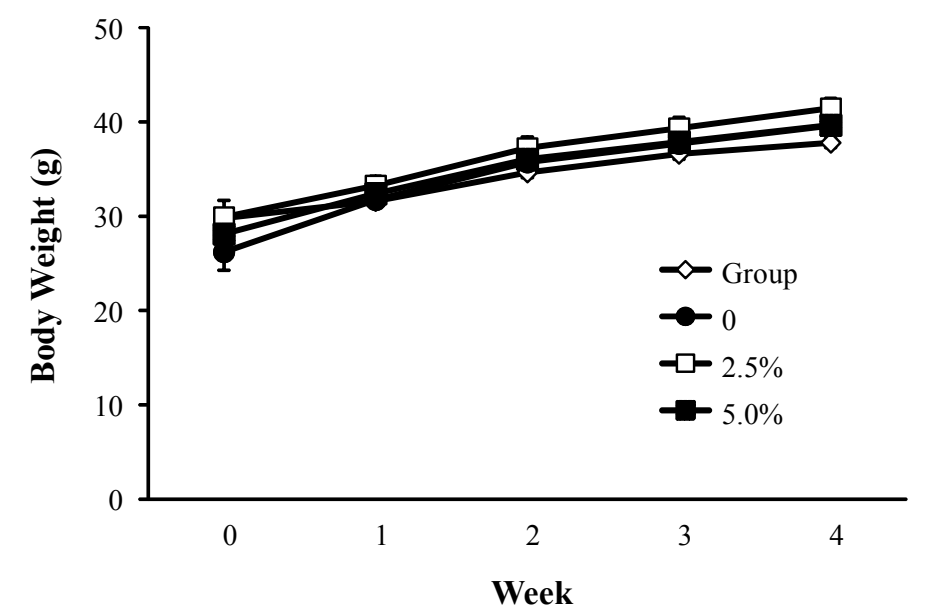

Figure 1. Effect of rice flour on body weight (Mean \pm S.E. $n=10)$. 


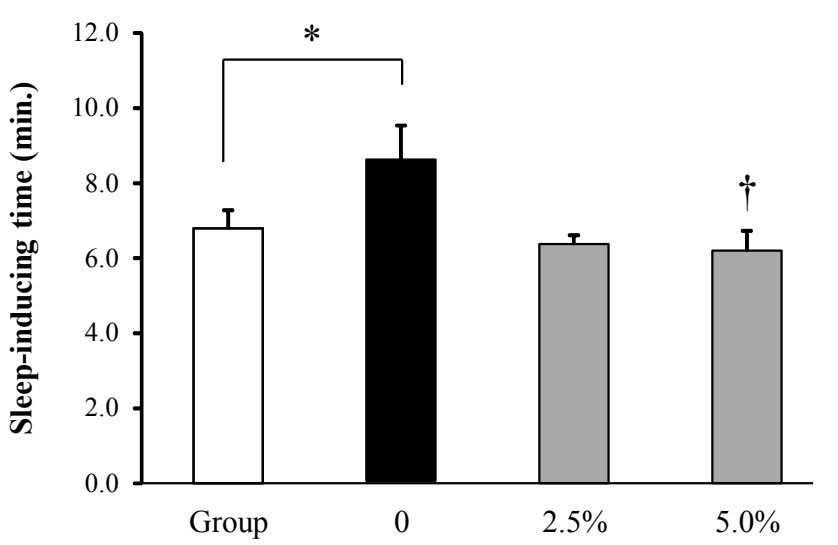

(a)

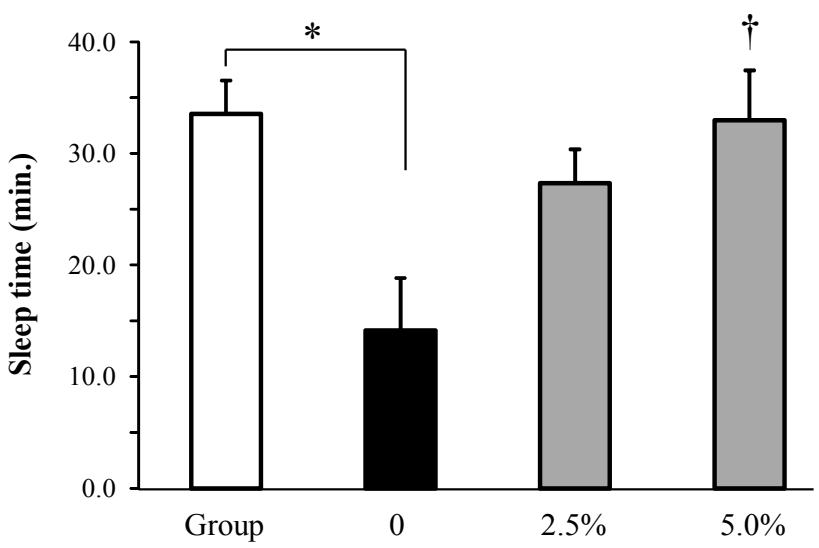

(b)

Figure 2. Effect of rice flour on the time to sleep introduction and the sleeping time induced by socially isolated stress in socially isolated mice (a) Time to sleep introduction, (b) Sleeping time (Mean \pm S.E. $n=10,{ }^{*}$ : $\mathrm{p}<0.05$ vs. Group, $\dagger$ : $p<0.05$ vs. 0 ).

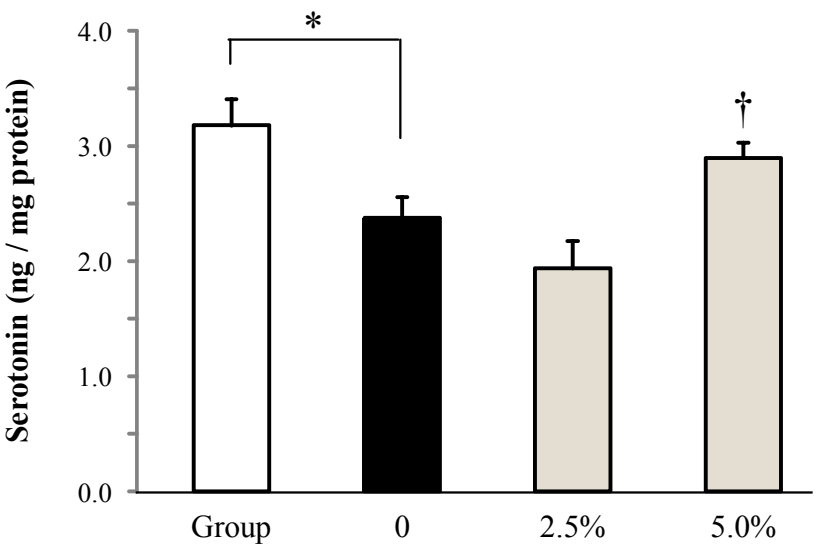

(a)

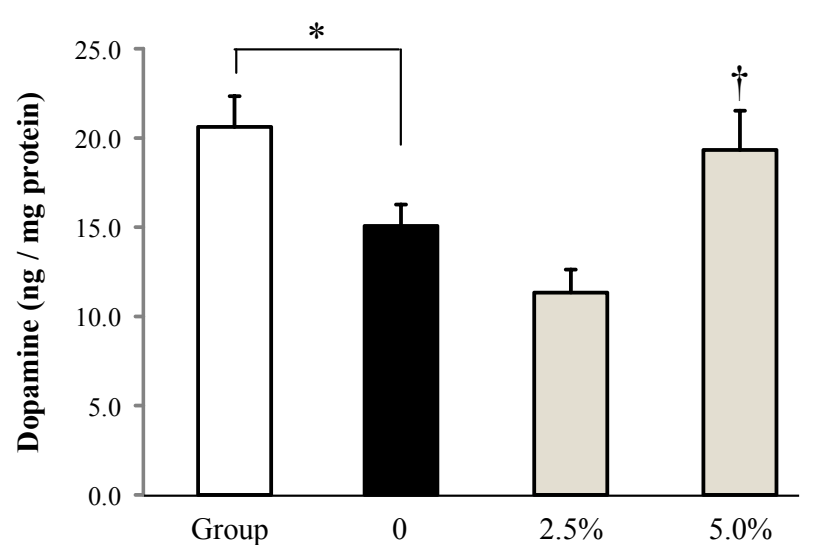

(b)

Figure 3. Effect of rice flour on serotonin and dopamine content in the frontal cortex of socially isolated mice (a) serotonin, (b) dopamine (Mean \pm S.E. $n=10,{ }^{*}: p<0.05$ vs. Group, $\dagger: p<0.05$ vs. 0 ).

\section{Discussion}

The amelioration of sleeping alleviates fatigue and stress at an early point and it is a major problem to have a healthy daily life. In the present study, we discussed the effect of rice flour on sleeping disorder induced by socially isolated stress. Rice flour was administrated at a dose of $2.5 \mathrm{w} / \mathrm{w} \%$ and $5.0 \mathrm{w} / \mathrm{w} \%(\mathrm{n}=10$ each) to a mouse reared in isolation for 4 weeks. Rice flour dose-dependently improved the extension of the time to sleep introduction and the shortening of sleeping hours induced by social isolation stress. Furthermore, blood corticosterone significantly increased by social isolation stress, and the increase significantly decreased by rice flour. It was confirmed that the amount of serotonin and dopamine in the prefrontal cortex significantly decreased by social isolation stress, and the decrease dose-dependently and significantly increased by rice flour. Finally, it was confirmed that IL-6 in the brain stem significantly increased by social isolation stress, and the increase dose-dependently and significantly decreased by rice flour. 


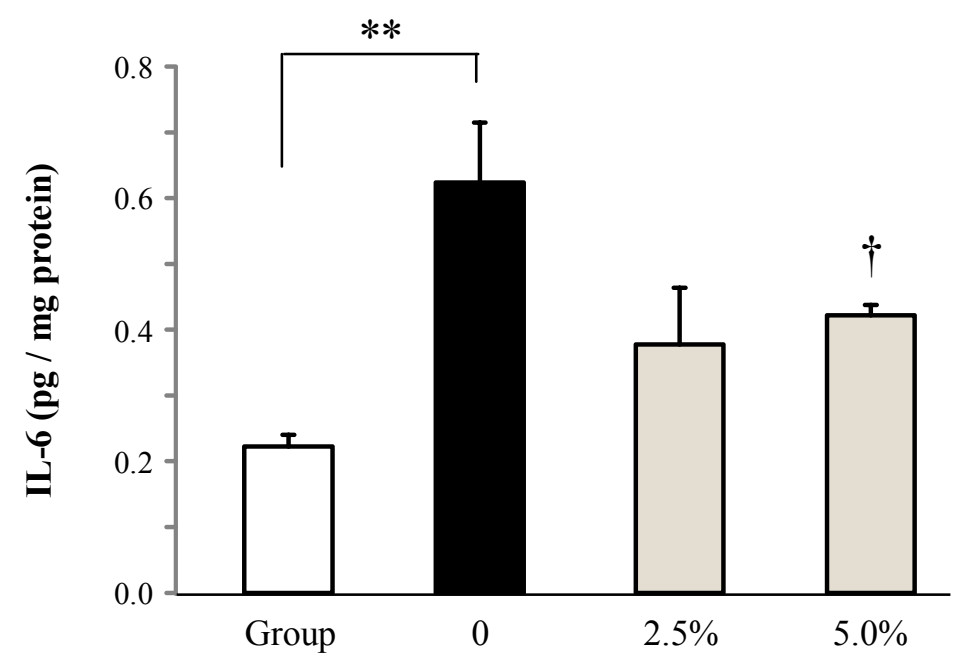

Figure 4. Effect of rice flour on the amount of IL-6 in the brainstem in socially isolated mice (Mean \pm S.E. $n=10,{ }^{*}: \mathrm{p}<0.05$ vs. Group, $\dagger: \mathrm{p}<0.05$ vs. 0).

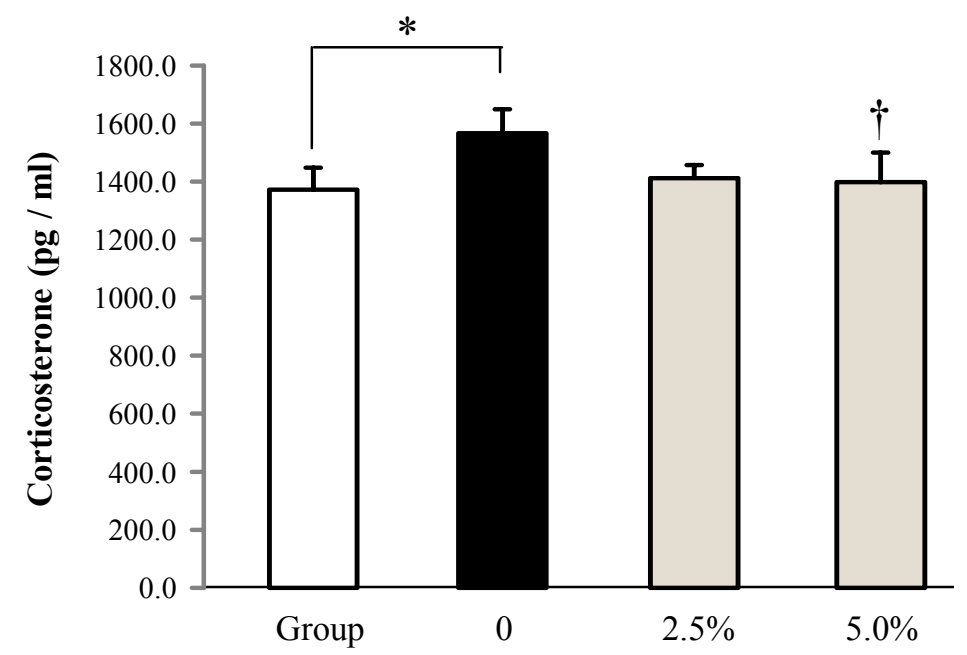

Figure 5. Effect of rice flour on blood serum corticosterone in socially isolated mice (Mean \pm S.E. $n=10,{ }^{*}: \mathrm{p}<0.05$ vs. Group, $\dagger: \mathrm{p}<0.05$ vs. 0 ).

We have reported that social isolation stress induces emotional disorder and develops sleeping disorder at an early point [4]. The sleep enhancing mechanism consists of nerve systems, such as serotonin in the brain, dopamine, noradrenaline and choline. Moreover, social isolation stress generically decreases the amount of dopamine, serotonin and glutamic acid in the prefrontal cortex [5]. It is unknown at present how social stress induces the decrease of monoamine in the brain. However, it is highly possible the promotion of the production and release of glucocorticoid (the main component of which is cortisol in humans and corticosterone in rodents) by stress stimulation is involved. The glucocorticoid receptor stimulation in the prefrontal cortex increases the release of stress-related neuromodulator dopamine [6] in the prefrontal cortex, through the positive-feedback system to midbrain dopamine neurons [7]. The present 
results demonstrated that serum glucocorticoid was significantly increased 4 weeks after the isolated rearing. Such chronic increases of glucocorticoid may rather induce adapted decreases of dopamine release in the prefrontal cortex in the long run. It is also reported that the serotonin release in the prefrontal cortex decreases as well by isolation stress [5], thus it is consistent with the present results. However, the relationship between corticosterone and sleep is still unclear.

The abnormality of the secretion rhythm of melatonin, which is sleeping hormone produced in the brain, is considered as a cause of sleeping disorder. Melatonin is synthesized from serotonin $\mathrm{N}$-acetyltransferase and hydroxyindole O-methyl transferase through serotonin. Although further verification is needed, it is suggested that rice flour increased serotonin and enhanced melatonin synthesis, therefore it improved sleep disorder. Brain monoamines are deeply involved in controlling emotions and cognitive behaviors, and a decrease of these neuromodulators would result in the abnormality of emotions and cognitive behaviors [8]. In the present study, rice flour recovered the amount of serotonin significantly decreased by stress. Although further verification is needed, it is suggested that consuming rice has an effect on controlling emotions and cognitive behaviors.

The relationship between stress and cytokine is regarded as important in recent years [9]. IL-6 is produced by astrocytes and microglia in the brain [10] and is known as an inflammatory marker cytokine for schizophrenia, depression, anxiety disorder, and mood disorders caused by chronic stress [9] [11]. According to the recent findings, IL- 6 regulates the activity of ERK (extracellular signal-regulated kinases) which is an important second messenger regulating neuronal plasticity, and IL-6 indeed decreases hippocampal LTP and cortical synaptic transmission [12]. Therefore, the increase of inflammatory factor level in the prefrontal cortex may decrease the activity of this cortical area, which might result in an increased emotional behavior controlled by the amygdala.

In the present study, rice flour decreased inflammatory cytokine, IL-6, which increased by stress. It was indicated the possibilities that rice flour has effects not only on the central action but also on the systemic symptoms caused by stress, although further research seems to be required regarding the mechanisms.

\section{Conclusion}

It is confirmed that rice flour has an amelioration effect on the shortening of the time to sleep introduction and the shortening of sleeping hours. It is also confirmed that it enhances catecholamine in the brain which decreases by stress. The present study demonstrated that rice flour is effective on sleeping disorder, and it also has a preventive effect on stress. Rice has long been the staple food for Japanese, and it is the representative proteins which can be obtained safely and securely. It is considered possible that daily diet leads us to good sleep.

\section{Conflicts of Interest}

The authors declare no conflicts of interest regarding the publication of this paper. 


\section{References}

[1] Chrousos, G.P. (2009) Stress and Disorders of the Stress System. Nature Reviews Endocrinology, 5, 374-381. https://doi.org/10.1038/nrendo.2009.106

[2] Mishima, K. (2012) Sleep Disorders and Sleep Medicine in Demented Patients. Sychiatria et Neurologia Japonica, 114, 167-170.

[3] Yoneyama, S., Sakurai, M., Nakamura, K., Morikawa, Y., Miura, K., Nakashima, M., Yoshita, K., Ishizaki, M., Kido, T., Naruse, Y., Nogawa, K., Suwazono, Y., Sasaki, S. and Nakagawa, H. (2014) Associations between Rice, Noodle, and Bread Intake and Sleep Quality in Japanese Men and Women. PLOS ONE, 9, e105198. https://doi.org/10.1371/journal.pone.0105198

[4] Koga, N., Yamaguchi, T., Lee, K.K. and Kobayashi, H. (2014) Kososan, a Standardized Traditional Japanese Herbal Medicine, Reverses Sleep Disturbance in Socially Isolated Mice via $\mathrm{GABA}_{\mathrm{A}}$-Benzodiazepine Receptor Complex Activation. Phytomedicine, 21, 697-703. https://doi.org/10.1016/j.phymed.2013.10.008

[5] Fone, K.C.F. and Porkess, M.V. (2008) Behavioral and Neurochemical Effects of Post-Weaning Social Isolation in Rodents-Relevance to Developmental Neuropsychiatric Disorders. Neuroscience and Behavioral Reviews, 32, 1087-1102. https://doi.org/10.1016/j.neubiorev.2008.03.003

[6] Lapiz-Bluhm, M.D. (2014) Impact of Stress on Prefrontal Glutamatergic, Monoaminergic and Cannabinoid Systems. In: Pariante, C.M. and Lapiz-Bluhm, M.D., Eds., Behavioral Neurobiology of Stress-related Disorders, Springer-Verlag, Berlin, Heidelberg, 45-66. https://doi.org/10.1016/j.neubiorev.2008.03.003

[7] Tonhajzerova, I., Ondrejka, I., Javorka, K., Turianikova, Z., Farsky, I. and Javorka, M. (2010) Cardiac Autonomic Regulation Is Impaired in Girls with Major Depression. Progress in Neuro-Psychopharmacology \& Biological Psychiatry, 34, 613-618. https://doi.org/10.1016/j.pnpbp.2010.02.023

[8] Suri, D., Teixeira, C.M., Cagliostro, M.K., Mahadevia, D. and Ansorge, M.S. (2015) Monoamine-Sensitive Developmental Periods Impacting Adult Emotional and Cognitive Behaviors. Neuropsychopharmacology, 40, 88-112. https://doi.org/10.1038/npp.2014.231

[9] Anisman, H. (2002) Stress, Immunity, Cytokines and Depression. Acta Neuropsychiatrica, 14, 251-261. https://doi.org/10.1034/j.1601-5215.2002.140601.x

[10] Gruol, D.L. (2014) IL-6 Regulation of Synaptic Function in the CNS. Neuropharmacology, 96, 42-45. https://doi.org/10.1016/j.neuropharm.2014.10.023

[11] Miller, B.J., Buckley, P., Seabolt, W., Mellor, A. and Kirkpatrick, B. (2011) Meta-Analysis of Cytokine Alterations in Schizophrenia: Clinical Status and Antipsychotic Effects. Biological Psychiatry, 70, 663-671. https://doi.org/10.1016/j.biopsych.2011.04.013

[12] Kitagaki, H., Hiyama, H., Kitazawa, T. and Shiohara, T. (2014) Psychological Stress with Long-Standing Allergic Dermatitis Causes Psychodermatological Conditions in Mice. Journal of Investigative Dermatology, 134, 1561-1569. https://doi.org/10.1038/jid.2014.31 